\title{
Importance of rheology in the prediction of structural transition phenomena in geopolymer matrices loaded with phosphogypsum
}

\author{
N. Lahlou ${ }^{1}$, M. Ouazzani Touhami ${ }^{1}$, R. Hattaf ${ }^{2}$, R. Moussa ${ }^{2}$ \\ ${ }^{1}$ Department of Physics, Faculty of Sciences Ain Chock, Hassan II University, 20470 Casablanca, Morocco \\ ${ }^{2}$ Department of Chemistry, Faculty of Sciences Ain Chock, Hassan II University, 20470 Casablanca, Morocco \\ nouhalahlou10@gmail.com
}

\begin{abstract}
Through a purely experimental approach, we proceed here to the description of the rheological behavior of the geopolymer matrices in the fresh state according to one or other of the parameters characterizing their formulation. This consolidates the different physicochemical techniques usually used for their characterization. This comes to allow us especially the definition of drafts for the implementation of empirical laws ensuring a better follow-up in the elaboration of these materials or even more optimization in their formulation. This description also allows us to follow the structural transition phenomena from the fresh state to the hardened state. We are particularly interested in demonstrating the impact of the addition of Phosphogypsum on the rheological behavior of geopolymers or on their kinetics of setting.
\end{abstract}

\section{Keywords: Rheology, geopolymer, Phosphogypsium, empirical law, kinetics setting}

\section{Introduction}

In the field of materials sciences, several physicochemical techniques are used for their elaboration or their structural transition (Thermo gravimetric analysis, X-ray diffraction ...). Since about fifty years, other macroscopic analyzes are introduced, it is essentially the methods of description based on the rheological behavior of the fresh state of these materials when it exists [1] [2]. In this context and in the light of the literature, it should be noted that this approach is taking on an increasingly important dimension, notably through the consolidation of control protocols for the improvement of formulation and exploitation of the considered materials. This concerns in particular the field of building materials [3].

In this work, we consider the particular case of aluminosilicate powders activated by alkaline solutions based on sodium and charged with phosphogypsum; waste from the phosphate industry. We are interested in highlighting the impact of the evolution of their rheological behavior, as a function of their structural properties, on the dynamics of their transition from the fresh state to the hardened state. The emphasis is on the characterization of the effects due to alkaline activation on the one hand or the addition of phosphogypsum in the geopolymer matrix on the other hand.

\subsection{Raw materials}

For the elaboration of geopolymer matrices, we consider the particular case where the components are:

- Sodium hydroxide pellets of purity $98.5 \%$

- An alkaline solution of sodium silicate with $45 \%$ by weight of dry matter and an $\mathrm{SiO}_{2} / \mathrm{Na}_{2} \mathrm{O}$ molar ratio of $2 \pm 0.1$

- An artificial pozzolan (Metakaolin) "ARGICAL-M $1000 "$. It is an amorphous, noncrystallized material with a small amount of quartz as the impurity phase. (figure 1)

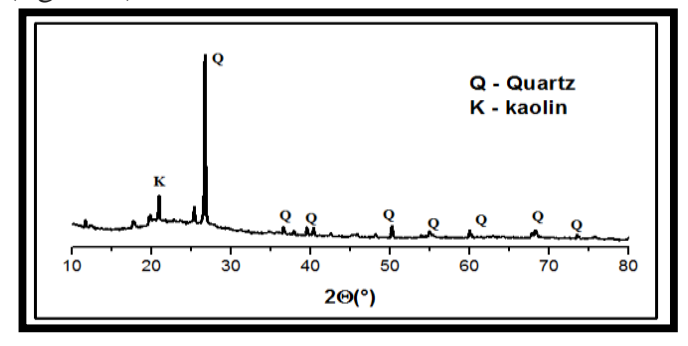

Figure 1: Metakaolin DRX

- A Phosphogypsum powder; waste from the phosphates industry in Morocco whose predominant phase is gypsum. (Figure 2)

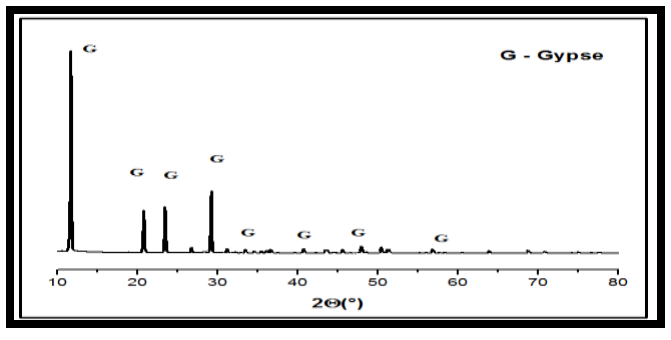

Figure 2: Phosphogypsum DRX

\subsection{Protocol of elaboration:}

Phosphogypsum-filled geopolymer paste is obtained from the following steps:

1- Preparation of a quantity $\mathrm{Q}_{1}$ of the sodium activation solution with a percentage $\mathrm{X}\left(=\% \mathrm{Na}_{2} \mathrm{O}\right)$ in predefined sodium oxide. The relation "X $=100 /\left(\mathrm{R}_{1}+\mathrm{R}_{2}+1\right)$ ", valid for $100 \mathrm{~g}$ of commercial solution, stops the values of the ratios $\mathrm{R}_{1}=\mathrm{SiO}_{2} / \mathrm{Na}_{2} \mathrm{O}$ and $\mathrm{R}_{2}=\mathrm{H}_{2} \mathrm{O} / \mathrm{Na}_{2} \mathrm{O}$ and allows to integrate at least, on the plane theoretical, a set of cases for which $R_{1}+R_{2}$ remains constant.

2- Addition of a quantity $\mathrm{Q}_{2}$ of the aluminosilicate powder (Metakaolin)

3- Addition of a quantity $\mathrm{Q}_{3}$ of phosphogypsum

\section{Raw materials and materials:}


4- Mixing the powders with the activation solution using a kneader for a time sufficient for the homogenization of the solution.

\subsection{Materials and methods:}

The rotary rheometer used is a Haake Rheostress 1 from "Thermo Scientific" operating at imposed speed or imposed stress and driven by Rheowin software. The tests are carried out in plane-plane geometry (cell PP60). For the tests carried out within the framework of this work, two configurations are retained:

a- A quasi-stationary configuration for monitoring the rheological behavior of these solutions.

b- An oscillatory configuration for the determination of the loss factors $G$ 'and conservation $G^{\prime}$ '

For the first case, a triangular rotation signal is imposed on the moving part of the rheometer with a rise time of $300 \mathrm{~s}$ and a maximum rotation value of $0.53 \mathrm{rad} / \mathrm{s}$, and for the analysis of the results only readings corresponding to the descending phases.

For the second case, the evolutions of $G$ 'and $G$ "are taken from a frequency sweep [from 1 to $20 \mathrm{~Hz}$ ] in the case of an imposed torque (harmonic).

\section{Results and discussion:}

In this section, we highlight the effects due to the variations of the control parameters $\mathrm{X}, \mathrm{Y}=\mathrm{Q}_{1} /\left(\mathrm{Q}_{2}+\right.$ $\left.\mathrm{Q}_{3}\right)$ and $\mathrm{Z}=\mathrm{Q}_{3} /\left(\mathrm{Q}_{2}+\mathrm{Q}_{3}\right)$ on the rheological behavior of the geopolymer matrices in order to have means capable of enabling:

$>$ Optimization of formulations or processes of elaboration of geopolymer matrices

$>$ The monitoring and control of changes in structural transitions within this matrix.

$>$ Approach prediction for improving the quality of finished products.

\subsection{Rheological characterization of the alkaline activation solution}

In view of the importance of the impact of alkaline activation on the evolution of geopolymers [4], we first verify that when $X$ varies, the behavior remains Newtonian and we present the empirical relations allowing to better understand the evolution of these solutions according to this parameter. (Figure.3.a.b).

$\eta=9.10^{-5} x^{5}-0,0071 x^{4}+0,227 x^{3}-3,5901 x^{2}+28,112 x-87,144$

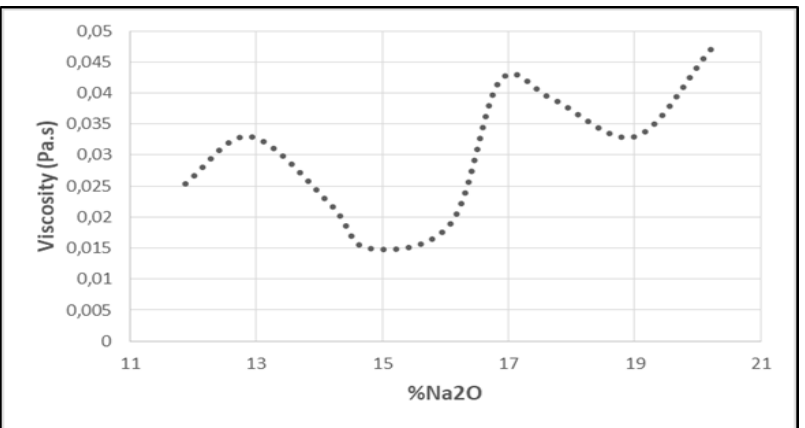

Figures 3.a: The evolutions of the viscosity versus the percentage of $\mathrm{Na}_{2} \mathrm{O}$
$G^{\prime}=-0,2443 x^{3}+11,427 x^{2}-174,82 x+883,21$

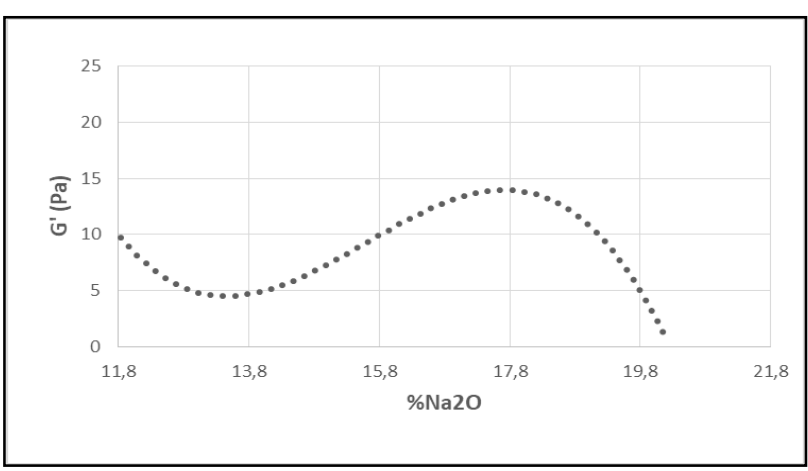

Figures 3.b: The evolutions of the conservation modulus versus the percentage of $\mathrm{Na}_{2} \mathrm{O}$

\subsection{Characterization of the rheological behavior of geopolymer matrices:}

For the fresh geopolymer matrices, we have been able to verify that the behavior remains described by the Herschel-Bulkley model in all the situations considered (figures 4.5), with apparent viscosities strongly impacted by the variations of $\mathrm{X}, \mathrm{Y}$ and $\mathrm{Z}$ and decreasing with the shear rate (shear-thinning behavior):

$$
\tau=\tau_{0}+\mathbf{k}(\dot{\boldsymbol{\gamma}})^{\mathbf{n}}
$$

It is a viscoplastic fluid with a yield stress $\tau_{0}$, consistency $\mathrm{k}$ and melt index $\mathrm{n}$.

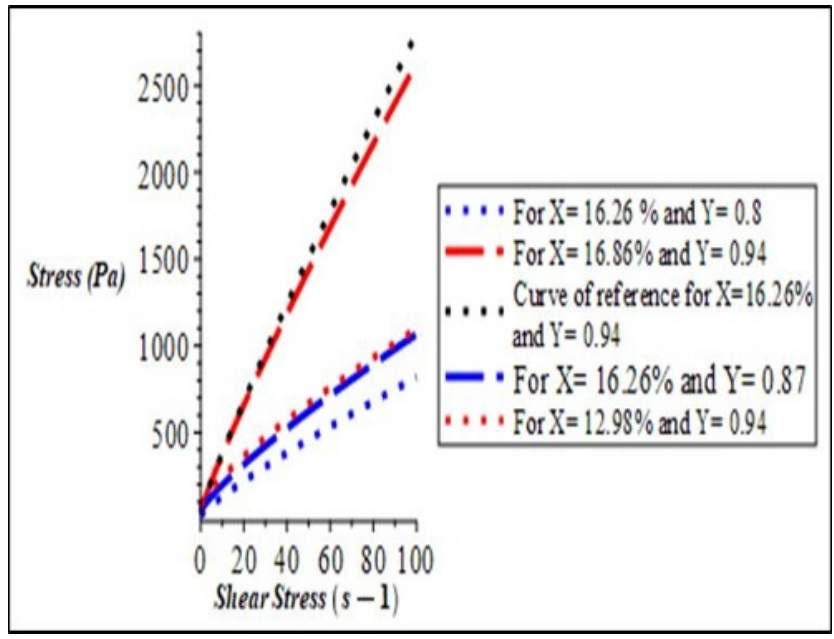

Figure 4: The effect of the parameters $X$ and $Y$ on the rheological behavior

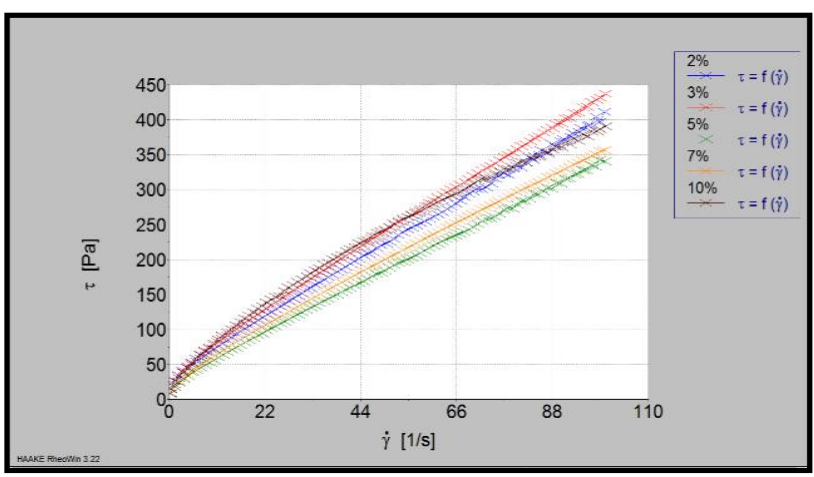

Figure5: The effect of $Z$ on the rheological behavior 
On the other hand, it should be noted that the loss and conservation parameters are, as shown in Figure (6), strongly affected by variation in $\mathrm{Z}$.
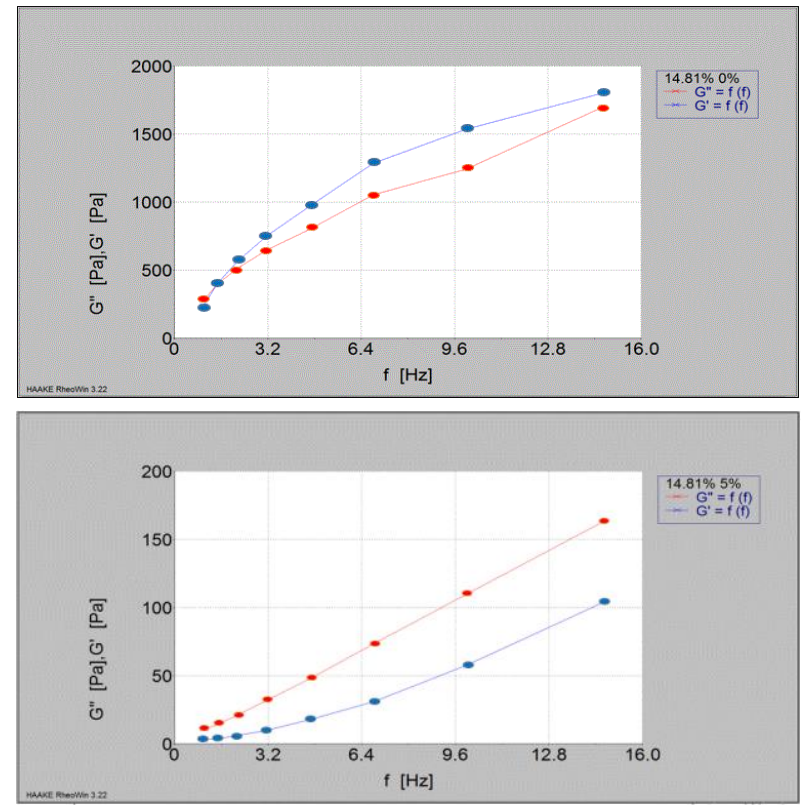

Figure 6: Evolution of the viscoelastic parameter for $X=14.81 \%, Z=0$ and $5 \%$

The correlation between these effects and changes in the control parameters is likely to consolidate the formulation processes of these matrices

\subsection{Impact of structural parameters on the transition phenomena:}

First, we highlight the effect of the different variations on the kinetics of setting (Figure.7.8). This structural transition demarcates the start of the condensation process and also makes it possible to set the operating or transport modalities of the geopolymers in the fresh state [3].

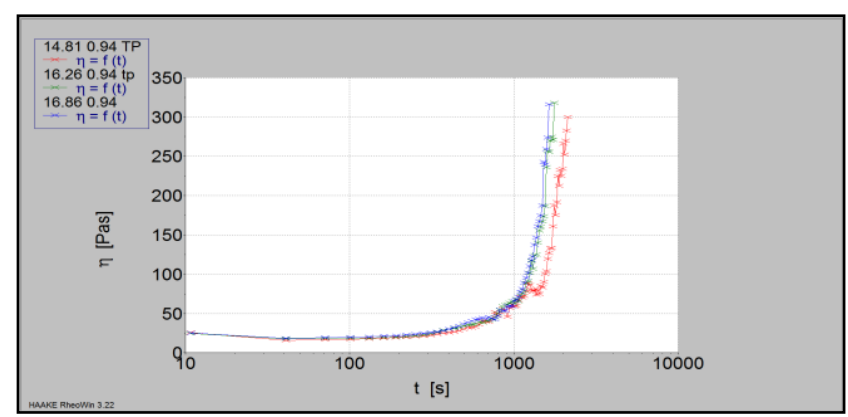

Figure 7: Impact of $X$ on the kinetics setting

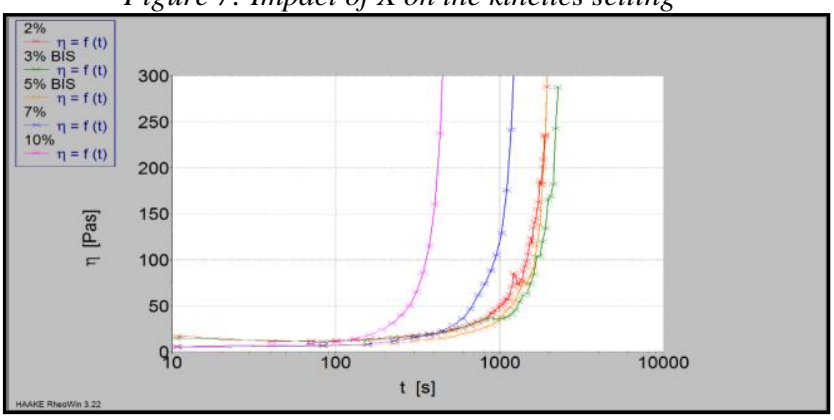

Figure 8: Impact of $Z$ on the kinetics setting
For the following and by way of example, we highlight in detail the effect of the incorporation of Phosphogypsum on the rheological parameters of the solution on the one hand and on its setting time on the other hand; the formulation chosen for this purpose corresponding to $\mathrm{X}=16.26 \%, \mathrm{Y}=0.94$ and $\mathrm{Z}$ varying from 0 to $10 \%$. We first observe that the parameters $\left(\tau_{0}\right.$, $\mathrm{k}$ and $\mathrm{n}$ ) are more or less affected by this addition; the evolutions of $\mathrm{k}$ and $\mathrm{n}$, with almost constant levels for $\mathrm{Z}<5 \%$, respectively increase or decrease abruptly from this value. It should be noted that for this value, the hardened state also has a maximum mechanical strength as it is reported in [5]. This result is in adequacy with that which one can raise from fig. 9 where the maximum setting time is obtained for a Phosphogypsum percentage close to $5 \%$.

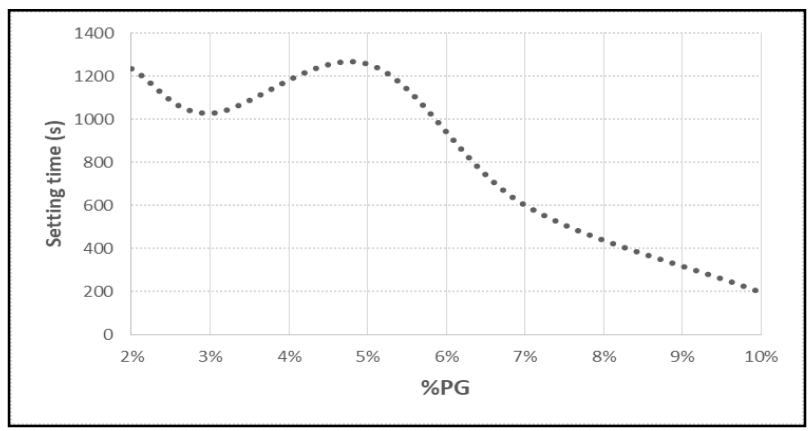

Figure 9: Evolution of the setting time for a different $Z$

\section{Conclusion:}

This work is part of a process to control and monitor the development process and improvement of operating protocols for geopolymer matrices. This is broken down in particular by:

- The introduction of empirical laws for the monitoring of the evolution of rheological variables according to the structural properties.

- Exploit properties related to the kinetics of setting for the definition of optimal value of these parameters.

Particular emphasis has been placed on determining the \% PP for which the setting time is the longest.

\section{References}

\section{https://cmm2019.sciencesconf.org/}

[1] P. Steins, Influence des paramètres de formulation sur la texturation et la structuration des géopolymères Thèse, École doctorale Sciences et Ingénierie des Matériaux, Mécanique, Energétique et Aéronautique, 2014.

[2] A. Bourlon. Physico-chimie et rhéologie de géopolymères frais pour la cimentation des puits pétroliers, Thèse, Université Pierre et Marie Curie, 2010.

[3] A. Aboulayt, M. Riahi, M. Ouazzani Touhami, H. Hannache, M. Gomina, R. Moussa, Properties of métakaolin based geopolymer incorporating calcium carbonate, J. Advanced Powder Technology,2017

[4] Wang J. W., Cheng T.W., Production geopolymer materials, Proceedings of the 7th International Symposium on East Asian Resources Recycling Technology (10-14 November 2003, Tainan, Taiwan), Tainan, 2003, 263-266

[5] AM Rashad, Potential use of phosphogypsum in alkaliactivated fly ash under the effects of elevated temperatures and thermal shock cycles, Journal of Cleaner Production, 2014. 